\title{
RELACIONES DE PODER EN LA SOCIEDAD PATRIARCAL ${ }^{1}$
}

\section{Ana Lucía Villarreal ${ }^{2}$}

El concepto de relaciones de fuerza o de poder ha sido ampliamente desarrollado desde los clásicos de la Sociología, como Marx y Weber, hasta autores como Poulantzas y Gramsci, con acento en lo económico, en la lucha de clases o lo político/ideológico, pero siempre en el marco de la vida pública.

En efecto, estos desarrollos sobre las relaciones de poder $\circ$ relaciones de dominación/subordinación han quedado divorciados de la vida cotidiana, y sobre todo de las relaciones intergenéricas, las cuales son objeto de estudio de la perspectiva de género. De esta forma se comete un nuevo acto de violencia contra las mujeres, en este caso por omisión, al eliminar o invisibilizar las relaciones de poder que se dan en otros espacios, por ejemplo en la vida privada.

Este artículo se inscribe dentro de la perspectiva de género, lo cual:

significa reconocer que las mujeres están situadas en la encrucijada entre producción y reproducción, entre la actividad económica y el cuidado y atención de los seres humanos y por lo tanto entre el crecimiento económico y el desarrollo humano. Ellas son las trabajadoras de ambas esferas: las más responsables y por lo tanto las que arriesgan más, las que sufren cuando las dos esferas funcionan de manera opuesta, y las más conscientes de la necesidad de una mejor integración entre las dos (Sen, 1995, p. 60).

\footnotetext{
${ }^{1}$ Este artículo fue construido como parte de la investigación: "Relaciones de poder. Mujeres en la encrucijada entre el trabajo productivo y el trabajo reproductivo", presentada en 1999, en la Maestría Centroamericana en Sociología de la Universidad de Costa Rica.

${ }^{2}$ M.Sc. en Sociología, Bachiller y Licenciada en Ciencias de la Comunicación Colectiva, UCR. Profesora de la Escuela de Ciencias de la Comunicación Colectiva UCR y de la Universidad Estatal a Distancia. Investigadora y Coordinadora del Programa de Investigación en Educación y Género del IIMEC, Facultad de Educación, UCR. Comunicadora en los campos de las Relaciones Públicas, de la Extensión Agrícola y de la Educación.
} 
Y, aunque Foucault con su "Microfísica del Poder" plantea que las relaciones de poder permean a todas las organizaciones y elabora ciertos aspectos que pueden ser de interés para el estudio de determinadas facetas de la vida privada; es la Teoría de Género la que más claramente establece que las relaciones de poder se presentan en todos los ámbitos de la sociedad, con lo cual las ubica tanto en la vida pública como en la privada. Así cruza el umbral y enciende una luz que permite analizar las relaciones en el ámbito de la vida cotidiana de la pareja y de la familia.

No obstante, a pesar del desarrollo de conceptos como el de relaciones de poder que se ha logrado desde el feminismo, todavía es poco el conocimiento que se tiene sobre la concreción de estas relaciones dominación/subordinación en la vida privada y sobre los factores que pueden eventualmente incidir para variar dichas relaciones.

Aunque se plantea que en las relaciones de poder quienes participan no carecen totalmente de poder, no se desglosa cuáles son los poderes con que cuentan las mujeres, más bien se profundiza en sus roles como subordinadas y se cae casi en una victimización, lo cual impide visualizar sus poderes -aunque limitados- para replantear dichas relaciones.

Partiendo de la reflexión anterior y con base en una amplia revisión bibliográfica sobre el concepto "relaciones de poder", tan llevado y traído, se elaboran las siguientes proposiciones, con el propósito de que sirvan como un eslabón más en el proceso humano del conocimiento.

Las relaciones de poder que se desarrollan en la sociedad patriarcal capitalista son de dominación/subordinación entre los géneros

Al partir de que la sociedad en que vivimos corresponde a una sociedad patriarcal capitalista $^{3}$, estamos asumimos el planteamiento que en ese sentido hace la autora Zillah

\footnotetext{
${ }^{3}$ Por patriarcado se entiende: la organización jerárquica masculina de la sociedad y, aunque su base legal institucional aparecía de manera mucho más explícita en el pasado, las relaciones básicas de poder han permanecido intactas hasta nuestros días. El sistema patriarcal se mantiene, a través del matrimonio y la familia, mediante la división sexual del trabajo y de la sociedad. El patriarcado tiene sus raíces en la biología más que en la economía o la historia. Las raíces del patriarcado se encuentran ya manifiestas a través de la fuerza y el control masculino en los propios yoes reproductivos de las mujeres. La definición de la mujer en esta estructura de poder no se define en términos de la estructura económica de clasi no en términos de la organización patriarcal de la sociedad (Eisenstein. 1977, p. 88-89).
} 
Eisenstein en el texto "Hacia el desarrollo de una teoría del patriarcado capitalista y el feminismo socialista". Y con ello también asumimos que las relaciones de poder que se desarrollan son de dominación/subordinación de lo masculino sobre lo femenino. Así como que estas relaciones se dan en todos los niveles de la sociedad: en los ámbitos de la vida privada y de la vida pública.

Estas relaciones de poder atraviesan todo el tejido social y se operacionalizan en cada campo de acción, de acuerdo con las reglas del juego que la sociedad ha definido. "Los poderes de dominio son sociales, grupales y personales, permiten explotar y oprimir a personas y grupos y todo tipo de colectividades. Se concretan en procesos concatenados de formas de intervenir en la vida de otras/os desde un rango y una posición de superioridad (valor, jerarquía, poderío)" (Lagarde, 1997, p. 69-70).

A la pregunta de qué es el poder, Foucault responde que es una relación de fuerzas, y amplía diciendo que el poder no es una forma, por ejemplo la forma Estado, sino que es una fuerza. Así como que la fuerza nunca está en singular, ya que su característica fundamental es estar en relación con otras fuerzas, de suerte que toda fuerza ya es relación, es decir, poder (Deleuze, 1987, p. 98).

En esta relación de fuerzas los hombres desarrollan relaciones de opresión mientras que las mujeres responden con subordinación, situación que se da a nivel macro y micro, a nivel de la vida pública como de la vida privada.

Estas relaciones dominación/subordinación se complementan con factores como los que plantea Foucault en el sentido de que: el poder no es esencialmente represivo (puesto que "incita, suscita, produce"); se ejerce más que se posee; pasa por los dominados tanto como por dominantes (puesto que pasa por todas las fuerzas en relación). Lo cual hace que su lectura no sea tan sencilla como parece.

\section{El poder es productivo}

Según Foucault (1981, p. 137) lo que hace que el poder se sostenga, que sea aceptado, es sencillamente que no pesa sólo como potencia que dice no, sino que cala de hecho, produce cosas, induce placer, forma saber, produce discursos; hay que considerarlo como una red 
productiva que pasa a través de todo el cuerpo social, en lugar de como una instancia negativa que tiene por función reprimir.

Esta afirmación (de que las relaciones de poder son productivas) es cuestionada por la teoría feminista, en el sentido de que esa productividad es positiva si se mide desde los parámetros definidos como tales por la sociedad patriarcal, pero no lo es para la mitad de la población que responde a relaciones de dominación con la subordinación, como forma de respuesta que le ha sido impuesta.

Es importante definir ¿para quién y en qué medida esas relaciones de poder son productivas? y ¿cuáles son los mecanismos de resistencia que desarrollan los individuos?.

Una posible respuesta a las preguntas anteriores las da la teoría feminista, cuando una de sus exponentes habla de la relación entre patriarcado y capitalismo:

En la medida en que el interés por la ganancia y por el control social se encuentren inextricablemente relacionados, el patriarcado y el capitalismo serán un proceso integral... El capitalismo usa al patriarcado y el patriarcado está determinado por las necesidades del capital... el patriarcado proporciona la organización sexual jerárquica de la sociedad necesaria para el control político, y en tanto que sistema político no se puede reducir a su estructura económica; mientras que el capitalismo como sistema económico de clase, impulsado por la búsqueda de ganancias, alimenta al orden patriarcal. Juntos forman la economía política de la sociedad (Eisenstein, 1977, p. 102-103).

Foucault $(1990,34)$ abre una ventana para leer esas relaciones cuando afirma que "no son unívocas; definen innumerables puntos de enfrentamiento, focos de inestabilidad, cada uno de los cuales comporta sus riesgos de conflicto, de luchas y de inversión por lo menos transitoria de las relaciones de fuerzas". Ventana que es cerrada al aclarar que este tipo de relaciones conlleva el conflicto y la inversión transitoria de las relaciones de fuerza, con lo cual hay que subrayar lo de transitoria, o sea que quienes asumen el dominio durante la relación, lo mantienen, aunque estas relaciones se inestabilicen. 
Estas afirmaciones confirman el punto de partida de que las relaciones de poder están marcadas por la sociedad patriarcal.

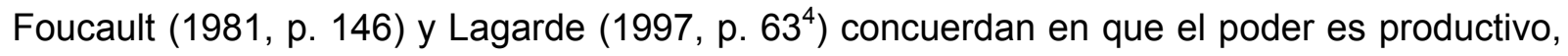
no obstante el primero no especifica para quién, mientras que la segunda claramente afirma que en la sociedad patriarcal capitalista el poder es productivo para los hombres. $Y$, en que en las relaciones de poder las personas que participan tienen poder:

En términos genéricos el poder es una relación de imposición de voluntad del dominante sobre el dominado $y$ es también influencia mutua. Las relaciones de dominación/subordinación son ineludibles, forman parte de la sociedad y de todas las relaciones interpersonales. A su vez que el dominante no está absolutamente determinado, no carece de libertad ni de espontaneidad, porque él hace parte de la totalidad de la relación e influye en el dominador, así sea en forma parcial (Maldonado, 1994, p. 149 - 151).

Las autoras y el autor citado coinciden en que quienes participan en las relaciones de poder no carecen de poder, que tanto el dominador como el dominado o subordinado poseen poderes, pero no especifican en qué consisten esos poderes, ni cómo inciden estos en las relaciones. Este es un elemento que es fundamental desarrollar en todos los niveles, tanto teórico como empírico, ya que de lo contrario se puede estar distorsionado el estudio de las relaciones de poder, porque se está sobrecargando la balanza en el platillo de quien domina y se invisibiliza la carga que tiene la persona dominada.

Al respecto Bertaux-Wiame (1985) señala nuevos senderos para el descubrimiento de la vida familiar, por medio de conocer el rol de la acción de las mujeres. Por ejemplo plantea que al ser partícipes en la producción de estatus social familiar, las mujeres desempeñan un rol fundamental en la transformación de las clases populares en clases medias, traduciendo cada día en la práctica las aspiraciones familiares para ellas y para sus hijos.

\footnotetext{
${ }^{4}$ Lagarde plantea que la explotación económica de las mujeres es fuente de poderío para los hombres y todas las personas e instituciones que se benefician y obtienen ganancias de la extracción de trabajo, valor, servicios y bienes de las mujeres; así como que el trabajo de las mujeres contribuye al incremento y desarrollo de aspectos y áreas básicas de la economía, la sociedad, la cultura y del sistema político.
} 
En los estudios de género este aspecto es fundamental ya que ello implica conocer los poderes, aunque limitados, que poseen las mujeres, los cuales pueden ser una clave para la construcción de relaciones equitativas y de por lo tanto de respeto.

Las relaciones de poder implican dependencia de quienes participan, porque están obligados a relacionarse entre sí, unos poseen lo que los otros no tienen

El término "relaciones de poder" implica de hecho que se da entre dos o más personas, quienes establecen dicha conexión porque necesitan satisfacer alguna necesidad. Esto implica a su vez que ambas personas aportan algo, unos poseen lo que las otras no tienen.

Entre quienes participan en una relación se pueden dar relaciones de diferentes tipo: de igualdad y equidad, de dominación y de subordinación. En cualquier tipo siempre quienes participan aportan algo que es importante para el otro, por lo tanto tienen una cuota de poder, la cual se sustenta en el valor que la/el donante y la/el receptor le den a su propio aporte; valor que se elabora de acuerdo a los parámetros de la sociedad, en este caso de la sociedad patriarcal.

Por lo tanto en las relaciones de poder se dan relaciones de dependencia y por pequeño que sea el aporte de una persona en esa relación, sin este la relación no existe. O sea, que quienes participan en una relación tienen poder y en mayor o menor grado dependen de esa relación para satisfacer alguna necesidad o deseo.

Lagarde (1997, p. 69) plantea que ideológicamente, quien está bajo dominio, es presa de la esperanza de satisfacer sus necesidades vitales y de obtener bienes vitales de los que carece.

Las relaciones que se dan en una familia son de dependencia, tanto depende el hombre del aporte de la mujer en los oficios domésticos, como la mujer de los recursos que el hombre aporta para la manutención económica de la familia.

Quien domina lo hace con la carga de poderío y de su posesión exclusiva de bienes vitales para quien está bajo su dominio, por eso son las necesidades y dependencia características de esta relación. La relación de obtener esos bienes genera dependencia en quien está bajo sujeción, pero es una 
dependencia vital, porque implica la necesidad de la presencia de quien domina, de sus bienes y de la relación (Lagarde, 1997, p. 70).

Es cierto (no se conoce con certeza hasta qué punto) que las mujeres amas de casa dependen económicamente de sus parejas para sobrevivir, pero a la vez esta afirmación de Lagarde desconoce (o por lo menos omite) el aporte que las mujeres le brindan a los hombres para que ellos puedan desempeñarse en el trabajo productivo, en la vida pública.

Al dársele mucho valor al aporte económico del hombre como proveedor de la familia, y basar en este las relaciones de dominación/subordinación en que viven las mujeres en la sociedad patriarcal, se está cayendo en varias trampas: reproducir la invisibilización del trabajo reproductivo; desconocer que en las relaciones de poder quienes participan tienen algún tipo de poder; reproducir el modelo de que en la sociedad lo que vale es aquello que se pueda valorar monetariamente; y sobre todo, hacer una lectura desde el patriarcado.

Al respecto existen otras versiones de este tipo de relaciones, como la que hace Gardiner al afirmar que "los hombres han sido vencidos más que las mujeres por la cultura de la dependencia, en el sentido de su dependencia de puestos de trabajo y de las mujeres para que los atiendan" (1993, p. 167).

El trabajo reproductivo en el patriarcado capitalista es la forma por excelencia en que la sociedad oprime e invisibiliza a las mujeres

Las relaciones de poder dominación/subordinación se materializan en el división vida privada/vida pública, y en la asignación de estos espacios a las personas según su género.

Por ello el que mujeres crucen el umbral de sus hogares para incorporarse al trabajo remunerado es un fenómeno que debe estudiarse. "Uno de los cambios importantes que se ha dado en la sociedad de las últimas décadas ha sido el ingreso de las mujeres al mercado laboral" (Lagarde, 1997, p. 51).

La explotación económica de las mujeres, al no pagarse el trabajo reproductivo, es base de su explotación erótica, reproductiva, afectiva, intelectual y cultural. Es fuente, en 
consecuencia, de poderío para los hombres y todas las personas (aún mujeres), y las instituciones que se benefician y obtienen ganancias de la extracción de trabajo, valor, servicios y bienes de las mujeres. A su vez la sociedad se beneficia, porque a través de su trabajo y de otras actividades, las mujeres contribuyen al incremento y desarrollo de aspectos y áreas básicas de la economía, la sociedad, la cultura y del sistema político.

Con respecto al trabajo de las mujeres en la sociedad es importante mencionar los siguientes datos: aunque las mujeres constituyen el $50 \%$ de la humanidad, trabajan $2 / 3$ partes de todas las horas trabajadas, reciben $1 / 3$ parte de los salarios pagados, son dueñas sólo del $1 \%$ de la propiedad mundial, sólo ocupan el $11 \%$ de los puestos parlamentarios en el mundo y el $7 \%$ de los puestos ministeriales (Sagot, 1995, p. 18).

Visto desde sus implicaciones globales las mujeres producen riqueza económica y social, preservan el medio, el territorio, la casa y el hogar, la familia, la pareja y las redes de parentesco, comunitarias, contractuales y políticas. A través de su cuerpo y de su subjetividad las mujeres gestan y dan vida a lo largo de sus vidas a las personas. $\mathrm{Y}$, con sus cuidados vitales, contribuyen a mantener la existencia día a día (Lagarde, 1997, p. 63).

A pesar de lo apuntado anteriormente, los patrones sociales vigentes en la sociedad actual no consideran que las actividades que realizan las mujeres sean históricas o trascendentales; por el contrario, se las ideologiza como instinto, amor, entrega, cuidados naturales, iluminación, labores propias de su sexo, no hacer nada.

En el campo económico, la falsa dicotomía (público/privado) ha significado que se han hecho suposiciones inexactas en cuanto a la producción económica, ignorando tanto el subsidio incalculable que la reproducción social le da a la producción, como las limitaciones de tiempo (y salud) que esto impone sobre las mujeres, que lo llevan a cabo restringiendo su participación en actividades públicas (Ashworth, 1995, p. 7).

En la sociedad patriarcal capitalista las mujeres poseen el "poder oculto" que es la otra cara del "poder legítimo".

Como se plantea en el punto anterior, las relaciones de poder que se desarrollan en la sociedad patriarcal implican dependencia de quienes participan en ellas. Ya se ha planteado 
la dependencia vital de la mujer de los ingresos del esposo, pero no se plantea la dependencia - también vital - que tiene el hombre de los servicios que la mujer presta por medio del trabajo reproductivo.

Aunque no se ha profundizado en este aspecto, se afirma que las mujeres poseen un poder oculto, el cual se construye en la dependencia del hombre del trabajo reproductivo. Razonamiento que es abordado por los teóricos del intercambio, quienes proponen que las relaciones de poder en la familia están en función de la contribución relativa de los esposos al matrimonio y en el valor de esas contribuciones. En la familia tradicional la mujer intercambia servicios domésticos, atención y cuidado de la prole y otra serie de labores, por soporte económico y protección del esposo (Szinovacz, 1984, p. 184).

Al invisibilizar la sociedad el aporte de la población femenina encasillándolo en el marco de la vida privada, se plantea que las mujeres poseen un poder oculto, el cual es la contraparte del poder de dominación genérica, tiene la limitación de no contar con la legitimación del reconocimiento social y funciona como contrapeso equilibrante que perpetúa la distribución actual del poder, que no es otra cosa que una distribución sexual del poder.

Dicho poder posee las siguientes características (Coria, 1991, p. 120):

- Lo ejercen las mujeres.

- Emerge desde un espacio oculto o semioculto, situado en el ámbito privado y doméstico.

- Utiliza recursos muy distintos a aquellos utilizados en el ejercicio del poder público, derivados de los sentimientos, de los afectos, de la contigüidad corporal, del erotismo.

- Sabe a hogar.

- Huele a afectos.

- Palpa cuerpos y proximidades.

- Adquiere el color nebuloso de lo que se oculta detrás de un vidrio oscuro.

- Tiene la textura escurridiza que tiene la legitimidad de lo marginal.

- Genera vinculaciones tortuosas (fácil blanco de posteriores reacciones hostiles).

- Fatalmente reedita comportamientos de sumisión en los que quedan atrapados, tanto quienes lo ejercen como quienes lo padecen.

- Es uno de los mayores obstáculos que desde la propia subjetividad femenina interviene en las mujeres condicionando su exclusión del poder público.

- Es una trampa al servicio del patriarcado. 
De la misma manera que la dependencia económica margina a las mujeres de los lugares de decisión, la adhesión consciente o inconsciente a este llamado "poder oculto" las excluye de los lugares legítimos de poder (Coria, 1991, p. 128).

Del planteamiento de Coria, se retoma su afirmación de que el "poder oculto" es la otra cara de la moneda del "poder masculino" y que juntos conforman las relaciones de poder de la sociedad actual, así como que para variar uno de ellos se debe variar el otro, y que este estado de cosas incide negativamente en el ser humano, para mujeres y hombres.

Tal vez uno de los retos del Feminismo sea conocer a profundidad cómo funciona ese poder oculto, sacarlo de la invisibilidad en que lo ubica la sociedad patriarcal y con base en ese conocimiento retomar y promover el desarrollo de sus valores, de los recursos que utiliza (muy distintos a aquellos utilizados en el ejercicio del poder público).

Al hacer visible el poder oculto se estará rompiendo una de las fuentes de poder de dominación, cual es el invisibilizar a las mujeres y desvalorizar lo que sucede en la vida privada. Esta tarea consistirá en hacer una lectura del poder femenino desde lo femenino, dejar de leerlo desde los valores masculinos, definidos como genéricos por el patriarcado y que desvalorizan lo femenino por definición.

Para variar las relaciones de poder dominación /subordinación en la sociedad patriarcal capitalista se deben establecer relaciones de cooperación, igualdad y equidad

Para variar las relaciones de poder de la sociedad actual es necesario romper con las relaciones como se han definido y desarrollado hasta la fecha y que corresponden a la sociedad patriarcal capitalista. Para ello se retoma la propuesta feminista de construir una sociedad con igualdad de derechos, con equidad para todas y todos sus integrantes y con reconocimiento y respeto de las diferencias.

Dicha sociedad se debe construir con base en (Lagarde, 1997, p. 38):

- Una redistribución de los poderes sociales.

- La transformación de los mecanismos de creación y reproducción de esos poderes, para deconstruir la opresión y la enajenación de género. 
- Crear poderes democráticos, de construcción de procesos para mejorar la calidad de vida de mujeres y hombres.

- Desarrollar opciones sociales dignas y una cultura que se corresponda con el nuevo paradigma, que pone en el centro lo humano compuesto por las mujeres y los hombres, la igualdad y la equidad como los principios de las relaciones de género y la construcción de calidad de vida y libertad.

- La distribución de poderes para vivir y preservar el mundo y para enriquecer la cultura con el derecho a la igualdad en la diversidad.

Según una de las corrientes del feminismo, el objetivo primordial de este movimiento fue y sigue siendo "La constitución de un espacio verdaderamente común a hombres y mujeres" (Collin, 1994, p. 317-318). Para lograrlo se recurre a las teorías de la igualdad. Pero esta igualdad debe entenderse como igualdad de derechos, no como igualdad de identidades, que por lo demás, se hará en provecho de la identidad masculina ya existente. Debe dejar lugar al juego de las diferencias, individuales o colectivas, sin por ello predefinirlas y prejuzgarlas, calificándolas o descalificándolas.

La teoría Feminista propone la construcción de relaciones igualitarias, y por lo tanto no de relaciones de poder entre las personas (según las definiciones que hemos aportado sobre el concepto de poder), sino sobre la base de que la sociedad debe crear las condiciones para que cada quien ejerza su poder para desarrollar sus capacidades individuales, no para dominar o subordinarse a otro.

Al aceptar que las relaciones de poder son de dominación/subordinación y que por lo tanto son dos caras de la misma realidad, superar estas relaciones implica construir relaciones de cooperación, con lo cual estaremos dejando en el pasado el ser o dominadores o dominados. "Sin la autonomía de las mujeres tampoco es posible la libertad de los hombres, porque con la falta de autonomía en ellas, ambos quedan aprisionados en una red de dependencias mutuas" (Coria, 1991, p. 48).

Este proceso corresponde al "desconstruccionismo" derridiano, el cual consiste ante todo en invertir la jerarquía existente en un momento dado, para hacer evidente la dependencia de un término con respecto al otro; inversión que es llevada a cabo desde el interior del sistema desconstruido, gracias a una doble escritura que desaloja y replantea los conceptos para 
hacer surgir, de ese modo, un nuevo concepto, que ya no puede ser incluido en el régimen anterior (Macaya, 1992, p. 6)

Dicha desconstrucción puede corresponder a visibilizar las relaciones de poder dominación/subordinación, relaciones que se establecen entre hombres y mujeres en el marco de una sociedad patriarcal; haciendo visible la dependencia existente entre quienes participan en dicha relación; y, haciendo visible el hecho de que para construir otro tipo de relaciones (que ya no serán de poder) se debe plantear y desarrollar un nuevo concepto, que en este caso corresponde a relaciones de apoyo, solidaridad y cooperación entre las personas, sin importar su género, las cuales evidentemente se podrán desarrollar en el marco de una sociedad diferente a la patriarcal capitalista actual.

Sociedad que se construirá con el aporte de las diferentes personas, sin importar su género y que por el contrario se conciba al ser humano con una persona integral, o lo que es lo mismo, que cada persona se desarrolle en los campos que considere propios, sin hacer la división entre trabajo productivo y trabajo reproductivo, entre trabajos femeninos y trabajos masculinos, entre trabajos con valor económico y trabajos que carecen de dicho valor; y superando así la falsa dicotonomía entre vida privada y vida pública.

Se plantea así superar el modelo de pareja concebido sobre la base de una relación jerárquica, en donde la distribución estereotipada de roles convierte a cada miembro en rey absoluto de un feudo (ellos reyes del mundo y ellas reinas del hogar), nos transforma a hombres y mujeres en víctimas y victimarios porque cada uno queda a merced del otro en aquello que desconoce (Coria, 1991, p. 31).

Esta concepción de la pareja, sustentada en la separación estricta de los roles y en la afirmación de la jerarquía, genera condiciones de opresión mutua.

Al superar esta visión de mundo bipolar de bueno o malo, público o privado, masculino o femenino, también estaremos dejando de lado la aceptación de que somos victimarios o víctimas, con lo cual podremos asumir la responsabilidad de construir nuestros propios sueños, dejando de lado las justificaciones de que los demás no nos dejan, cada mujer debe asumir su vida, pasar de la inmovilidad que implica el percibirse como víctima para percibirse como protagonista responsable de su existencia. 
Pero para construir este mundo, es necesario conocer, visibilizar y valorar la cultura femenina que enfatiza los valores de cooperación, interdependencia, consideración y relaciones interpersonales expresivas; en vez de las relaciones instrumentales. Con lo cual se podría transformar la estructura jerárquica de las instituciones políticas existentes, tornándolas más sensitivas a las necesidades humanas, más flexibles, democráticas y personalizadas (Bystydzienski, 1995, p. 53-65).

Esta propuesta feminista representa una superación de aquella que en el pasado buscó develar la injusticia del poder masculino reclamando para la mujer el derecho a ejercer ese mismo poder, sin entrar a cuestionar los principios generales de poder y las escalas de valores que sustentan ese sistema dentro del cual demandan un papel protagónico. Reclamando así la participación de la mujer en el desempeño de roles tradicionalmente masculinos que gozan de un estatus elevado dentro de una concepción del mundo en que los bienes materiales y de consumo sustituyen a los valores de la integridad personal, el respeto y la consideración y búsqueda del bien común (Vega, 1994, p. 146).

Además, reconoce los avances de las mujeres al conquistar la vida pública y hacer sus propuestas desde la identidad femenina, no tratando de asumir como propios los parámetros y valores masculinos, definidos como genéricos por la sociedad.

Resumiendo, después de revisar las definiciones y desarrollos del concepto poder y relaciones de poder desde diferentes campos, como la sociología, la política y el feminismo, se retoma que dichas relaciones se desarrollan en el marco de la sociedad patriarcal, lo cual se apoya en el abordaje que sobre estos conceptos ha desarrollado el Feminismo, básicamente desde una de sus exponentes contemporáneas, como lo es Marcela Lagarde, así como la propuesta que sobre el poder hace Foucault.

Se retoma el hecho sustantivo de que el poder es central en las relaciones entre hombres y mujeres y define la forma y contenido de las mismas. Así como que el meollo del asunto está en la subordinación de las mujeres en las relaciones de poder y su exclusión de los ámbitos y espacios donde éste se ejerce y se define (García, 1997, p. 45). 
De esta forma se da una mirada visibilizando a las mujeres, rompiendo con las propuestas que desconocen la realidad de las diferencias de género que caracterizan las relaciones sociales.

Develando la otra mitad de la realidad, con lo cual se modifica la ya conocida, crea una nueva realidad y plantea nuevos problemas y nuevas alternativas.

Al mismo tiempo que se asume la propuesta feminista de crear una sociedad en la cual no existan relaciones de dominación/subordinación, sino que estas sean sustituidas por relaciones de apoyo, solidaridad, igualdad de derechos y respeto a las diferencias y a la diversidad.

De esta forma se valida la afirmación de Izquierdo (1991, p. 80) en el sentido de que:

El patriarcado no se puede superar haciendo patriarcas a quienes no lo son, para que todas tengan los derechos formales y reales del patriarca, sino superando ese orden social, con su régimen de derechos. Aquello a lo que se denomina derechos, en la práctica es un sistema de privilegios y el privilegio remite necesariamente a su otra cara, que es la desposesión. Derechos/privilegios para unes es desposesión para otras.

La educación reproduce las relaciones de poder dominación/subordinación La educación, como una de las instituciones de la sociedad, reproduce este tipo de relaciones en los diferentes ámbitos de la vida de sus actor@s.

Esta reproducción se da a todo nivel en la educación: en la sobrevaloración de los valores masculinos (agresividad, competencia, control, poder de decisión, razón, independencia, ser servido, etc.) y la desvalorización de los valores femeninos (cooperación, apoyo, interdependencia, emoción, pasividad, servicio, etc.). En la invisibilización del aporte milenario de las mujeres a la construcción y reproducción de la sociedad. En la valorización de la vida pública (trabajo productivo) y desvalorización de la vida privada (trabajo reproductivo). En fin, en el mantener y reproducir un mundo bipolar, en lugar de un mundo integrado, en el cual todas sus partes tienen funciones complementarias que cumplir. 
Uno de los mayores obstáculos para variar este tipo de relaciones es el que las personas las interiorizamos y nos apropiamos de ellas como parte del proceso de socialización, por lo cual las vemos como "lo normal" y como la única forma de relacionarnos con nosotr@s mism@s y con I@s demás.

Por ello, uno de los primeros pasos que debemos dar para variar este tipo de relaciones, si es que consideramos que debemos variarlas, es visibilizando cómo se operacionalizan en la práctica docente y aún más, en la vida cotidiana.

De esta forma podremos arriesgarnos a practicar otras formas de relacionarnos con las demás personas que participan en los procesos educativos; con ello estaremos construyendo otras formas de hacer las cosas y sobre todo estaremos actuando conscientemente y dejando de reproducir la sociedad patriarcal y sus relaciones, con las cuales los seres humanos que la integran actúan según el rol en que se encuentren (con poder/dominadores o sin poder/subordinados) y no como personas interdependientes, creativas, asertivas y propositivas.

De esta forma estaremos pasando de la creencia de que el poder de cada persona es externo a ella (cargo, títulos, bienes), es el poder de decidir en la vida de los demás; para participar en la construcción de personas cuyo poder sea interno, el cual se basa en una autoestima elevada, al ser coherentes entre lo que dicen y lo que hacen y al utilizar su poder para establecer relaciones con equidad y respeto a las diferencias.

\section{REFERENCIA}

Ashworth, Georgina. (1995). Gender and Governance. UNDP Gender in development program occasional report series. Traducción de Ana Cecilia Escalante y Sylvia Clar.

Bystydzienski, Jill. (1995). Cultura femenina en Noruega. En Espacios: Revista Centroamericana de Cultura Política No. 5. Friedrich Ebert Stiftung, FLACSO, CEDAL. Julio/setiembre.

Coria, Clara. (1991). El dinero en la pareja. Algunas desnudeces sobre el poder. Barcelona, España: Ediciones PAIDOS. 
Collin, Francoise. (1993). Diferencia y diferendo: La cuestión de las mujeres en filosofía. En: Historia de las mujeres. Historia de las mujeres en Occidente. El Siglo XX. (Tomo 5, pp. 291-321). Madrid: Taurus.

Deleuze, Gilles. (1987). Foucault. Barcelona, España: Paidós Studio.

Eisenstein, Zillah. (1984). Hacia el desarrollo de una teoría del patriarcado capitalista y el feminismo socialista 1977. En: Teoría Feminista. (Selección de textos). República Dominicana: Ediciones populares feministas.

Foucault, Michel. (1981). Verdad y poder. Diálogo con M. Fontana. En Un diálogo sobre el poder y otras conversaciones. Madrid, España: Alianza Editorial y Materiales.

(1990). Vigilar y castigar. Nacimiento de la prisión. México: Siglo XXI.

García, Ana Isabel; Gomáriz, Enrique. (1989). Mujeres Centroamericanas: efectos del conflicto. San José, Costa Rica: FLACSO.

García, Evangelina. (1997). Derechos políticos y ciudadanía de las mujeres: una vía género sensitiva y paritaria al poder y al liderazgo. 1 edición. San José, Costa Rica: Centro Nacional para el Desarrollo de la Mujer y la Familia.

Gardiner, Jean. (1975). El papel del trabajo doméstico. En El ama de casa bajo el capitalismo. Barcelona, España: Editorial ANAGRAMA.

Gindling, Tim. (1991). Ingresos de las mujeres y crisis económica en Costa Rica. Instituto de Investigaciones Económicas. Universidad de Costa Rica. Costa Rica.

(1997). Género y feminismo. Desarrollo humano y democracia. Cuadernos inacabados 25. España: Horas y Horas.

Izquierdo, Ma. Jesús. (1991). Un marco teórico para las relaciones de sexo y de género. En: Luna, Lola (Comp). Mujeres y sociedad. Nuevos enfoques teóricos y metodológicos. España: Universitat de Barcelona.

Lockwood, M. (1984). The women's movement and the family: a socio-historical analysis of contraitson social change. En: Women and the family. Two decades of change. London: Haworth Press.

Macaya, Emilia. (1992). Cuando estalla el silencio. Para una lectura femenina de textos hispánicos. San José, Costa Rica: Editorial de la Universidad de Costa Rica.

Maldonado Gómez, Ma. Cristina. (1994). Relaciones de dominación en la familia. En: Discurso, género y mujer. Compilado por Gabriela Castellanos y otras. Centro de Estudios de género y la manzana de la discordia. Editorial Facultad de Humanidades, Universidad del Valle. Cali, Colombia.

Sagot, Montserrat. (1995). Socialización de género, violencia y femicidio. En: Revista Reflexiones (412). Facultad de Ciencias Sociales, Universidad de Costa Rica. Diciembre. 
Sen, Gita. (1995). Una economía alternativa desde una perspectiva de género. Development: Journal of the Society for International Development (SID).

Szinovacz, M. (1984). Changing family roles and interactions. En Hess, B. y Sussman, M. (eds.). Women and the family. Wodewades of change. London: Haworth Press.

Vega, Isabel. (1994). Trabajo productivo de la mujer y cambio en los roles familiares. El caso de Costa Rica. En Anuario de Estudios Centroamericanos, 20 (1). Costa Rica.

Villarreal, Cecilia. (1992). La familia: ¿Ideal realizado o realidad idealizada? Revista Educación 16. Universidad de Costa Rica.

Weber, Max. (1969). Economía y sociedad: Esbozo de sociología comprensiva. México: Fondo de Cultura Económica. Primera reimpresión, tomo II.

Weber, Max. (1944). Economía y sociedad. Tomo I. La Habana, Cuba: Editorial de Ciencias Sociales, Instituto Cubano del Libro. 